\title{
LONGTIME DYNAMICS OF A CONDUCTIVE FLUID IN THE PRESENCE OF A STRONG MAGNETIC FIELD
}

\author{
C. BARDOS, C. SULEM AND P. L. SULEM
}

\begin{abstract}
We prove existence in the large of localized solutions to the MHD equations for an ideal conducting fluid subject to a strong magnetic field. We show that, for large time, the dynamics may reduce to linear Alfven waves.
\end{abstract}

1. Introduction. In contrast with a uniform velocity field, a uniform magnetic field has a significant dynamical effect on a conducting flow. This is easily seen by writing the (ideal) MHD equations:

$$
\begin{aligned}
& \frac{\partial v}{\partial t}+v \cdot \nabla v=-\nabla p+b \cdot \nabla b \\
& \frac{\partial b}{\partial t}+v \cdot \nabla b=b \cdot \nabla v \\
& \nabla \cdot v=\nabla \cdot b=0
\end{aligned}
$$

in terms of the Elsässer variables

$$
Z^{+}=v+b, \quad Z^{-}=v-b
$$

which satisfy

$$
\begin{aligned}
& \frac{\partial Z^{+}}{\partial t}+Z^{-} \cdot \nabla Z^{+}=-\nabla p, \\
& \frac{\partial Z^{-}}{\partial t}+Z^{+} \cdot \nabla Z^{-}=-\nabla p, \\
& \nabla \cdot Z^{+}=\nabla \cdot Z^{-}=0 .
\end{aligned}
$$

When equations (1.3) are linearized around the static solution with a constant magnetic field $B_{0}$, one obtains that the fluctuations $z^{ \pm}=Z^{ \pm} \mp B_{0}$ propagate along the $B_{0}$ magnetic field in opposite directions. This suggests that in the original nonlinear problem, a strong enough magnetic field will reduce the nonlinear interactions [1] and inhibit formation of strong gradients. This effect was observed in direct numerical simulations of equations (1.3) with periodic boundary conditions [2]. These calculations showed that in the presence of a strong enough magnetic field, solutions remain analytic in a strip whose width is bounded from below.

In this paper, we consider the problem in the entire $R^{2}$ or $R^{3}$; we prove that for large $B_{0}$ and small enough localized initial fluctuations $z^{ \pm}$, the solution of the MHD equations (1.3) remain smooth for all time and that the nonlinear interactions become asymptotically negligible when $t \rightarrow+\infty$.

Received by the editors March 11, 1986.

1980 Mathematics Subject Classification (1985 Revision). Primary 35Q20; Secondary 76E25. 
2. Equations and functional framework. The fluctuations $z^{ \pm}$satisfy

$$
\begin{aligned}
& \frac{\partial z^{+}}{\partial t}+Z^{-} \cdot \nabla z^{+}=-\nabla p, \\
& \frac{\partial z^{-}}{\partial t}+Z^{+} \cdot \nabla z^{-}=-\nabla p, \quad x \in R^{n}, n=2 \text { or } 3 \\
& \nabla \cdot z^{+}=\nabla \cdot z^{-}=0, \\
& z^{+}(x, 0)=z_{0}^{+}(x) ; \quad z^{-}(x, 0)=z_{0}^{-}(x) .
\end{aligned}
$$

As a consequence,

$$
j^{ \pm}=\operatorname{Curl} z^{ \pm}
$$

obeys

$$
\begin{aligned}
& \frac{\partial j^{+}}{\partial t}+Z^{-} \cdot \nabla j^{+}=-\sum_{k} \nabla z_{k}^{-} \wedge \partial_{k} z^{+}, \\
& \frac{\partial j^{-}}{\partial t}+Z^{+} \cdot \nabla j^{-}=-\sum_{k} \nabla z_{k}^{+} \wedge \partial_{k} z^{-} .
\end{aligned}
$$

Two families of characteristics are associated to equations (2.1), (2.3),

$$
\begin{array}{lll}
\dot{x}^{-}(t)=Z^{-}\left(x^{-}(t), t\right), & \text { and } & \dot{x}^{+}(t)=Z^{+}\left(x^{+}(t),(t)\right), \\
x^{-}(0)=a^{-}, & & x^{+}(0)=a^{+} .
\end{array}
$$

We define the operators $A^{+}$and $A^{-}$by

$$
\begin{aligned}
& A^{+} z^{+}(x, t)=f\left(x+B_{0} t\right) z^{+}(x, t) \\
& A^{-} z^{-}(x, t)=f\left(x-B_{0} t\right) z^{-}(x, t)
\end{aligned}
$$

where, for example,

$$
f(x)=1+|x|^{2} .
$$

Similar weights were introduced by Klainerman [3] in the context of nonlinear wave equations.

To justify the above definitions, we remind that, in the linear problem, $z^{ \pm}$and $j^{ \pm}$are just transported along the characteristics $\dot{x}^{\mp}=\mp B_{0}$. It follows that if the initial conditions are $z_{0}^{ \pm}(x)=\phi^{ \pm}(x) /\left(1+|x|^{2}\right)$, where $\phi^{ \pm}(x)$ are uniformly bounded, the quantities $A^{ \pm} z_{l}^{ \pm}(x, t)$ associated to the linear solutions $z_{l}^{ \pm}$are bounded uniformly in time. For the nonlinear problem, we have

$$
\begin{aligned}
\frac{d}{d t} A^{+} j^{+}\left(x^{-}(t), t\right)= & f\left(x^{-}(t)+B_{0} t\right) \frac{d}{d t} j^{+}\left(x^{-}(t), t\right) \\
& +z^{-}\left(x^{-}(t), t\right) \cdot \nabla f\left(x^{-}(t)+B_{0} t\right) j^{+}\left(x^{-}(t), t\right) \\
= & -f\left(x^{-}(t)+B_{0} t\right) \nabla z_{k}^{-} \wedge \partial_{k} z^{+}\left(x^{-}(t), t\right) \\
& +z^{-}\left(x^{-}(t), t\right) \cdot \nabla f\left(x^{-}(t)+B_{0} t\right) j^{+}\left(x^{-}(t), t\right) \quad \text { (from (2.3)). }
\end{aligned}
$$


Thus,

(2.8)

$$
\begin{aligned}
A^{+} j^{+} & \left(x^{-}(t), t\right) \\
= & A^{+} j_{0}^{+}\left(a^{-}\right)-\int_{0}^{t} \frac{1}{f\left(x^{-}(\tau)-B_{0} \tau\right)} A^{-} \nabla z^{-}\left(x^{-}(\tau), \tau\right) \wedge A^{+} \partial_{k} z^{+}\left(x^{-}(\tau), \tau\right) d \tau \\
& +\int_{0}^{t} \frac{1}{f\left(x^{-}(\tau)-B_{0} \tau\right)} A^{-} z^{-}\left(x^{-}(\tau), \tau\right) \\
& \cdot \frac{\nabla f\left(x^{-}(\tau)+B_{0} \tau\right)}{f\left(x^{-}(\tau)+B_{0} \tau\right)} A^{+} j^{+}\left(x^{-}(\tau), \tau\right) d \tau
\end{aligned}
$$

Similarly,

(2.9)

$$
\begin{aligned}
A^{+} z^{+}\left(x^{-}(t), t\right)= & A^{+} z_{0}^{+}\left(a^{-}\right)-\int_{0}^{t} f\left(x^{-}(\tau)+B_{0} \tau\right) \nabla p\left(x^{-}(\tau), \tau\right) d \tau \\
& +\int_{0}^{t} \frac{A^{-} z^{-}\left(x^{-}(\tau), \tau\right)}{f\left(x^{-}(\tau)-B_{0} \tau\right)} \cdot \frac{\nabla f\left(x^{-}(\tau)+B_{0} \tau\right)}{f\left(x^{-}(\tau)+B_{0} \tau\right)} A^{+} z^{+}\left(x^{-}(\tau), \tau\right) d t .
\end{aligned}
$$

Analogous equations hold for $A^{-} j^{-}\left(x^{+}(t), t\right)$ and $A^{-} z^{-}\left(x^{+}(t), t\right)$.

The analysis presented in this paper consists of showing that also, for the nonlinear problem, $A^{ \pm} z^{ \pm}(x, t)$ and $A^{ \pm} j^{ \pm}(x, t)$ remain uniformly bounded in $C^{0, \alpha}\left(R^{n}\right)$ under some smallness hypotheses on the initial conditions.

$C^{0, \alpha}\left(R^{n}\right)(0<\alpha<1)$ is the space of (vectorial) functions which are Hölder continuous. It is equipped with the norm

$$
|u|_{0, \alpha}=|u|_{0}+\sup _{x, y \in R^{n}} \frac{|u(x)-u(y)|}{|x-y|^{\alpha}}
$$

where

$$
|u|_{0}=\sup _{x \in R^{n}}|u(x)|
$$

$C^{1, \alpha}\left(R^{n}\right)$, which is the space of functions which are Hölder continuous together with their derivatives, is equipped with the norm

$$
|u|_{1, \alpha}=|u|_{0, \alpha}+\sum_{i=1}^{n}\left|\frac{\partial u}{\partial x_{i}}\right|_{0, \alpha} .
$$

We recall that, for initial conditions $z_{0}^{ \pm}$in $C^{1, \alpha}\left(R^{n}\right)$ with zero divergence, there exists, during a finite time $t<T_{*}$, a unique solution $\left(z^{+}, z^{-}\right)$in $C\left(0, T_{*}, C^{1, \alpha}\left(R^{n}\right)\right)$ [4]. Thus, if initially,

$$
\left|A^{+} z_{0}^{+}\right|_{1, \alpha},\left|A^{-} z_{0}^{-}\right|_{1, \alpha} \leq B_{0} / 4
$$

there exists a time $T_{1}$ such that for $t<T_{1},\left|A^{+} z^{+}\right|_{1, \alpha}$ and $\left|A^{-} z^{-}\right|_{1, \alpha}$ remain smaller than $B_{0} / 2$. 


\section{A Schauder-type lemma.}

LEMMA 3.1. For $z^{ \pm}$satisfying $\nabla \cdot z^{ \pm}=0$, and $j^{ \pm}=\operatorname{Curl} z^{ \pm}$we have

$$
\left|A^{ \pm} \nabla z^{ \pm}\right|_{0, \alpha} \leq C\left(\left|A^{ \pm} z^{ \pm}\right|_{0, \alpha}+\left|A^{ \pm} j^{ \pm}\right|_{0, \alpha}\right) .
$$

PROOF. The proof of this lemma is similar to that of the Schauder lemma [5]. We only consider the case of + signs. The other is identical. For simplicity we give the proof in three dimensions. The case of dimension two is very similar.

If $\nabla \cdot z^{+}=0$ and $j^{+}=\operatorname{Curl} z^{+}$, then $z^{+}$satisfies

$$
\Delta z^{+}=-\operatorname{Curl} j^{+} \text {. }
$$

In three dimensions, we define

$$
\begin{gathered}
K(|x|)=\left\{K_{k}(|x|)=\frac{1}{4 \pi} \nabla \partial_{k} \frac{\theta(|x|)}{|x|}\right\}_{k=1,2,3} \\
H(|x|) u=\frac{1}{4 \pi} \nabla \Delta \frac{1-\theta(|x|)}{|x|} \otimes u-(u \cdot \nabla) \nabla \otimes \nabla\left[\frac{1-\theta(|x|)}{|x|}\right]
\end{gathered}
$$

where $\theta$ is a $C^{\infty}$ function equal to 1 for $|x|<1$ and 0 for $|x|>2$. We write

$$
A^{+} \nabla z^{+}(x)=f\left(x+B_{0} t\right) \int K(|x-y|) \frac{A^{+} j^{+}(y)}{f\left(y+B_{0} t\right)} d y .
$$

Separating the contribution at the origin and at infinity, we write

$$
A^{+} \nabla z^{+}(x)=P(x)+Q(x)
$$

with

$$
P(x)=f\left(x+B_{0} t\right) \int K(|x-y|)\left[\frac{A^{+} j^{+}(y)}{f\left(y+B_{0} t\right)}-\frac{A^{+} j^{+}(x)}{f\left(x+B_{0} t\right)}\right] d y
$$

and

$$
Q(x)=f\left(x+B_{0} t\right) \int H(|x-y|) \frac{A^{+} z^{+}(y)}{f\left(y+B_{0} t\right)} d y .
$$

We have

$$
\begin{aligned}
P(x)= & \int K(|x-y|)\left(A^{+} j^{+}(y)-A^{+} j^{+}(x)\right) d y \\
& +\int K(|x-y|) A^{+} j^{+}(y) \frac{\left|f\left(x+B_{0} t\right)-f\left(y+B_{0} t\right)\right|}{f\left(y+B_{0} t\right)} d y .
\end{aligned}
$$

Thus,

$$
|P(x)|_{0} \leq C\left|A^{+} j^{+}\right|_{0, \alpha} .
$$

On the other hand, denoting by $C$ various constants,

$$
\begin{aligned}
|Q(x)| \leq & C\left|A^{+} z^{+}\right|_{0} \int_{|x-y|>1} \frac{1}{|x-y|^{4}} \frac{f\left(x+B_{0} t\right)}{f\left(y+B_{0} t\right)} d y \\
& +C\left|A^{+} z^{+}\right|_{0} \int_{1<|x-y|<2} \frac{1}{|x-y|^{3}} \frac{f\left(x+B_{0} t\right)}{f\left(y+B_{0} t\right)} d y \\
\leq & C\left|A^{+} z^{+}\right|_{0} \int_{v>1} \frac{d v}{v^{2}} \frac{1+a^{2}}{1+(v-a)^{2}}+\int_{1<v<2} \frac{d v}{v} \frac{1+a^{2}}{1+(v-a)^{2}}
\end{aligned}
$$


where $a=\left|x+B_{0} t\right|$. In (3.10), the integrals

$$
\begin{aligned}
& \int_{v<1} \frac{d v}{v^{2}} \frac{1+a^{2}}{1+(v-a)^{2}}=\left[\frac{1}{v^{2}}+\frac{a}{a^{2}+1} \ln \frac{v^{2}}{1+(v-a)^{2}}\right. \\
&\left.+\frac{3 a^{2}-1}{a^{2}+1} \tan ^{-1}\left(1+(v-a)^{2}\right)\right]_{1}^{\infty} \\
& \int_{1<v<2} \frac{d v}{v} \frac{1+a^{2}}{1+(v-a)^{2}}=\left[\ln \sqrt{\frac{v}{1+(v-a)^{2}}}+2 a \tan ^{-1}(v-a)\right]_{1}^{2} \\
&= C+2 a \tan ^{-1} \frac{1}{1+(2-a)(1-a)}
\end{aligned}
$$

are uniformly bounded in $a$. It follows that

$$
|Q(x)|_{0} \leq C\left|A^{+} z^{+}\right|_{0} .
$$

We now turn to $\left(1 /|x-y|^{\alpha}\right)\left|A^{+} \nabla z^{+}(x)-A^{+} \nabla z^{+}(y)\right|$, and write $d=|x-y|$. If $d>$ 1 we bound separately the two terms of the difference. If $d<1$ in $|P(x)-P(y)| / d^{\alpha}$, we separate the contributions $\sigma_{p}$ and $\bar{\sigma}_{p}$ from $\Sigma=\{|x-u|<2 d\}$ and $R^{3} \backslash \Sigma$ respectively. For $\sigma_{p}$, we bound separately the two terms of the difference and get

$$
\sigma_{p} \leq C\left|A^{+} j^{+}\right|_{0, \alpha} .
$$

We write $\bar{\sigma}_{p}$ in the form

$$
\begin{aligned}
\bar{\sigma}_{p}= & \frac{f\left(x+B_{0} t\right)}{d^{\alpha}} \int(K(|x-u|)-K(|y-u|))\left[\frac{A^{+} j^{+}(u)}{f\left(u+B_{0} t\right)}-\frac{A^{+} j^{+}(x)}{f\left(x+B_{0} t\right)}\right] d u \\
& -\frac{f\left(y+B_{0} t\right)}{d^{\alpha}} \int(K(|y-u|))\left[\frac{A^{+} j^{+}(x)}{f\left(x+B_{0} t\right)}-\frac{A^{+} j^{+}(y)}{f\left(y+B_{0} t\right)}\right] d u \\
& +\left[\frac{f\left(x+B_{0} t\right)-f\left(y+B_{0} t\right)}{d^{\alpha}}\right] \int K(y-u)\left[\frac{A^{+} j^{+}(u)}{f\left(u+B_{0} t\right)}-\frac{A^{+} j^{+}(x)}{f\left(x+B_{0} t\right)}\right] d u .
\end{aligned}
$$

In the first integral of (3.15)

$$
\begin{aligned}
(K(|x-u|)-K(|y-u|)) & \leq c|x-y| \sup _{0 \leq \gamma \leq 1}|u-\gamma y-(1-\gamma) x|^{-4} \\
& \leq \frac{c}{2}|x-y||u-x|^{-4} .
\end{aligned}
$$

The first integral of (3.15) is thus bounded from above by $C\left|A^{+} j^{+}\right|_{0, \alpha}$. For the second integral, we write

$$
\int K(|y-u|) d u=\int_{|u-x| 2 d} \nabla\left[\frac{1}{|y-u|} \theta(|y-u|)\right] \wedge \vec{n} d \vec{\sigma} \leq C
$$

and thus get

$$
\frac{1}{d^{\alpha}}|P(x)-P(y)| \leq C\left|A^{+} j^{+}\right|_{0, \alpha} .
$$

On the other hand, for $d<1$,

$$
\frac{1}{d^{\alpha}}|Q(x)-Q(y)| \leq \sup _{x}\left|Q^{\prime}(x)\right|
$$


where

$$
\begin{aligned}
\left|Q^{\prime}(x)\right| \leq & C\left|A^{+} z^{+}\right|_{0} \int_{v>1} \frac{1}{v^{3}} \frac{1+a^{2}}{1+(v-a)^{2}} d v \\
& +C\left|A^{+} z^{+}\right|_{0} \int_{1<v<2} \frac{1}{v^{2}} \frac{1+a^{2}}{1+(v-a)^{2}} d v \\
& +2 C a\left|A^{+} z^{+}\right|_{0} \int_{v>1} \frac{1}{v^{2}} \frac{1}{1+(v-a)^{2}} d v \\
& +2 C a\left|A^{+} j^{+}\right|_{0} \int_{1<v<2} \frac{1}{v} \frac{1}{1+(v-a)^{2}} d v
\end{aligned}
$$

For the first two integrals of (3.19), we notice that for $|v|>1,1 / v^{3}<1 / v^{2}$ and $1 / v^{2}<1 / v$ and using (3.11) and (3.12), we obtain that they are uniformly bounded with respect to $a$. For the last two integrals, we use $1 \leq c\left(a^{2}+1\right)$ to recover previously computed integrals. Putting together the above estimates, we get (3.1).

4. A priori estimates. We define

$$
M^{ \pm}(t)=\sup _{0 \leq \tau \leq t}\left(\left|A^{ \pm} z^{ \pm}(\cdot, \tau)\right|_{0, \alpha}+\left|A^{ \pm} j^{+}(\cdot, \tau)\right|_{0, \alpha}\right)
$$

and

$$
N(t)=M^{+}(t)+M^{-}(t) .
$$

In this section, we establish a uniform bound for $N(t)$.

Proposition 4.1. For $t<T_{1}$ we have

$$
\begin{aligned}
& \left|A^{+} z^{+}(\cdot, t)\right|_{0, \alpha}+\left|A^{+} j^{+}(\cdot, t)\right|_{0, \alpha} \\
& \quad \leq\left(\left|f z_{0}^{+}\right|_{0, \alpha}+\left|f j_{0}^{+}\right|_{0, \alpha}\right) \exp \frac{M^{-}(t)}{2\left|B_{0}\right|-M^{-}(t)} .
\end{aligned}
$$

ProOF. It follows from (2.8) that

$$
\begin{aligned}
\left|A^{+} j^{+}(\cdot, t)\right|_{0} \leq & \left|A^{+} j_{0}^{+}\right|_{0}+\sup _{0 \leq \tau \leq t}\left(\left|A^{-} z^{-}(\cdot, \tau)\right|_{0}+\left|A^{-} \nabla z^{-}(\cdot, \tau)\right|_{0}\right) \\
& \cdot \int_{0}^{t} K\left(x^{-}(\tau), \tau\right)\left(\left|A^{+} z^{+}(\cdot, \tau)\right|_{0, \alpha}+\left|A^{+} j^{+}(\cdot, \tau)\right|_{0, \alpha}\right) d \tau
\end{aligned}
$$

where

$$
K\left(x^{-}(\tau), \tau\right)=\frac{1}{f\left(x^{-}(\tau)-B_{0} \tau\right)}\left[1+\frac{\left|\nabla f\left(x^{-}(\tau)+B_{0} \tau\right)\right|}{f\left(x^{-}(\tau)+B_{0} \tau\right)}\right]
$$

satisfies

$$
\int_{0}^{t} K\left(x^{-}(\tau), \tau\right) d \tau \leq c \int_{0}^{t} \frac{d \tau}{1+\left(x^{-}(\tau)-B_{0} \tau\right)^{2}}
$$

By the change of variables

$$
\xi(\tau)=\left(x^{-}(\tau)-B_{0} \tau\right) \cdot \frac{B_{0}}{\left|B_{0}\right|}=\left(a^{-}-2 B_{0} \tau+\int_{0}^{\tau} z^{-}\left(x^{-}\left(\tau^{\prime}\right), \tau\right) d \tau^{\prime}\right) \cdot \frac{B_{0}}{\left|B_{0}\right|}
$$


we have

$$
\begin{aligned}
\int_{0}^{t} K\left(x^{-}(\tau), \tau\right) d \tau & \leq c \int_{0}^{\infty} \frac{d \xi}{1+\xi^{2}} \frac{1}{\left|2 B_{0}\right|-\sup _{0 \leq \tau \leq t}\left|z^{-}(\cdot, \tau)\right|_{0}} \\
& \leq \frac{c}{\left|2 B_{0}\right|-\sup _{0 \leq \tau \leq t}\left|A^{-} z^{-}(\cdot, \tau)\right|_{0}} \leq \frac{c}{2\left|B_{0}\right|-M^{-}(t)}
\end{aligned}
$$

To estimate the $C^{0, \alpha}$-norms, we consider the distance $\rho(\tau)=\left|x^{-}(\tau)-y^{-}(\tau)\right|$ between two characteristics starting from $x^{-}(0)=a^{-}$and $y^{-}(0)=b^{-}$. We have

$$
\begin{aligned}
& \frac{d}{d t}\left[\frac{\left|A^{+} j^{+}\left(x^{-}(t), t\right)-A^{+} j^{+}\left(y^{-}(t), t\right)\right|}{\left|x^{-}(t)-y^{-}(t)\right|^{\alpha}}\right] \\
& \leq \alpha \frac{\left|\rho^{\prime}(t)\right|}{\rho(t)^{\alpha+1}}\left|A^{+} j^{+}\left(x^{-}(t), t\right)-A^{+} j^{+}\left(y^{-}(t), t\right)\right| \\
& \quad+\frac{1}{\rho(t)^{\alpha}}\left|\frac{d}{d t}\left(A^{+} j^{+}\left(x^{-}(t), t\right)-A^{+} j^{+}\left(y^{-}(t), t\right)\right)\right|
\end{aligned}
$$

Integrating from 0 to $t$ and using (2.8) we have

$$
\begin{aligned}
& \frac{\left|A^{+} j^{+}\left(x^{-}(t), t\right)-A^{+} j^{+}\left(y^{-}(t), t\right)\right|}{\rho(t)^{a}} \\
& \leq\left|A^{+} j_{0}^{+}\right|_{0, \alpha}+\alpha \int_{0}^{t} \frac{\left|\rho^{\prime}(\tau)\right|}{\rho(\tau)^{\alpha+1}}\left|A^{+} j^{+}\left(x^{-}(\tau), \tau\right)-A^{+} j^{+}\left(y^{-}(\tau), \tau\right)\right| d \tau \\
& +\mid \int_{0}^{t} \frac{d \tau}{\rho(\tau)^{\alpha}} \frac{A^{-} \nabla z_{k}^{-}\left(x^{-}(\tau), \tau\right)}{f\left(x^{-}(\tau)-B_{0} \tau\right)} \wedge A^{+} \partial_{k} z^{+}\left(x^{-}(\tau), \tau\right) \\
& -\frac{A^{-} \nabla z_{k}^{-}\left(y^{-}(\tau), \tau\right)}{f\left(y^{-}(\tau)-B_{0} \tau\right)} \wedge A^{+} \partial_{k} z^{+}\left(y^{-}(\tau), \tau\right) \\
& +\mid \int_{0}^{t} \frac{d \tau}{\rho(\tau)^{\alpha}} \frac{1}{f\left(x^{-}(\tau)-B_{0} \tau\right)} \frac{\nabla f\left(x^{-}(\tau)+B_{0} \tau\right)}{f\left(x^{-}(\tau)+B_{0} \tau\right)} \\
& \cdot A^{-} z^{-}\left(x^{-}(\tau), \tau\right) A^{+} j^{+}\left(x^{-}(\tau), \tau\right) \\
& -\frac{1}{f\left(y^{-}(\tau)-B_{0} \tau\right)} \frac{\nabla f\left(y^{-}(\tau)+B_{0} \tau\right)}{f\left(y^{-}(\tau)+B_{0} \tau\right)} \\
& \cdot A^{-} z^{-}\left(y^{-}(\tau), \tau\right) A^{+} j^{+}\left(y^{-}(\tau), \tau\right) \\
& \leq\left|A^{+} j_{0}^{+}\right|_{0, \alpha}+\alpha \int_{0}^{t} \frac{\mid \rho^{\prime}(\tau)}{\rho(\tau)^{\alpha+1}}\left|A^{+} j^{+}\left(x^{-}(\tau), \tau\right)-A^{+} j^{+}\left(y^{-}(\tau), \tau\right)\right| d \tau \\
& +C \sup _{0 \leq \tau \leq t}\left|A^{-} z^{-}(\cdot, \tau)\right|_{1, \alpha} \int_{0}^{t} K\left(x^{-}(\tau), \tau\right) \\
& \cdot\left(\left|A^{+} z^{+}(\cdot, \tau)\right|_{0, \alpha}+\left|A^{+} j^{+}(\cdot, \tau)\right|_{0, \alpha}\right) d \tau \\
& +C \sup _{0 \leq \tau \leq t}\left|A^{-} z^{-}(\cdot, \tau)\right|_{1, \alpha} \int_{0}^{t} \frac{1}{\rho(\tau)^{\alpha}}\left[H\left(x^{-}(\tau), y^{-}(\tau), \tau\right)\right] \\
& \cdot\left(\left|A^{+} z^{+}(\cdot, \tau)\right|_{0}+\left|A^{+} j^{+}(\cdot, \tau)\right|_{0}\right) d \tau
\end{aligned}
$$


wherein, for $0 \leq t \leq T_{1}$

(4.11)

$$
\begin{aligned}
& \int_{0}^{t} \frac{1}{\rho(\tau)^{\alpha}}\left|H\left(x^{-}(\tau), y^{-}(\tau), \tau\right)\right| d \tau \\
& \quad \leq \int_{0}^{t} \frac{1}{\rho(\tau)^{\alpha}}\left[\frac{1}{f\left(x^{-}(\tau)-B_{0} \tau\right)}-\frac{1}{f\left(y^{-}(\tau)-B_{0} \tau\right)}\right] \cdot\left[1+\frac{\left|\nabla f\left(x^{-}(\tau)+B_{0} \tau\right)\right|}{f\left(x^{-}(\tau)+B_{0} \tau\right)}\right] d \tau \\
& \quad+\int_{0}^{t} \frac{1}{\rho(\tau)^{\alpha}} \frac{1}{f\left(y^{-}(\tau)-B_{0} \tau\right)}\left[\left|\frac{\nabla f\left(x^{-}(\tau)+B_{0} \tau\right)}{f\left(x^{-}(\tau)+B_{0} \tau\right)}-\frac{\nabla f\left(y^{-}(\tau)+B_{0} \tau\right)}{f\left(y^{-}(\tau)+B_{0} \tau\right)}\right|\right] d \tau
\end{aligned}
$$

Let us denote by $P_{1}$ and $P_{2}$ the two integrals arising in the r.h.s of (4.11). For $\rho(\tau)<1$, we write

$$
\begin{aligned}
& P_{1}=\int_{0}^{t} \frac{1}{\rho(\tau)^{\alpha}}\left[\frac{1}{f\left(x^{-}(\tau)-B_{0} \tau\right)}-\frac{1}{f\left(y^{-}(\tau)-B_{0} \tau\right)}\right] \\
& \cdot\left[1+\frac{\mid \nabla f\left(x^{-}(\tau)+B_{0} \tau\right)}{f\left(x^{-}(\tau)+B_{0} \tau\right)}\right] d \tau \\
& \leq C \int_{0}^{t} \frac{\left|x^{-}(\tau)-B_{0} \tau+y^{-}(\tau)-B_{0} \tau\right|}{\left[1+\left(x^{-}(\tau)-B_{0} \tau\right)^{2}\right]\left[1+\left(y^{-}(\tau)-B_{0} \tau\right)^{2}\right]} \rho(\tau)^{1-\alpha} d \tau \\
& \leq C \int_{0}^{t} \rho(\tau)^{1-\alpha}\left[\frac{1}{f\left(x^{-}(\tau)-B_{0} \tau\right)}+\frac{1}{f\left(y^{-}(\tau)-B_{0} \tau\right)}\right] d \tau
\end{aligned}
$$

For $\rho(\tau)>1$, we bound separately the terms of the difference and get

$$
P_{1} \leq \frac{C}{2\left|B_{0}\right|-M^{-}(t)} \text {. }
$$

Similarly

$$
\begin{aligned}
P_{2}=\int_{0}^{t} \frac{1}{\rho(\tau)^{\alpha}} \frac{1}{f\left(y^{-}(\tau)-B_{0} \tau\right)} \frac{\left|x^{-}(\tau)+B_{0} \tau\right|-\left|y^{-}(\tau)+B_{0} \tau\right|}{1+\left(x^{-}(\tau)-B_{0} \tau\right)^{2}} \\
+\frac{\left|y^{-}(\tau)+B_{0} \tau\right| \rho(\tau)\left|x^{-}(\tau)+y^{-}(\tau)+2 B_{0} \tau\right|}{\left[1+\left(x^{-}(\tau)+B_{0} \tau\right)^{2}\right]\left[1+\left(y^{-}(\tau)+B_{0} \tau\right)^{2}\right]} d \tau
\end{aligned}
$$

which leads to

$$
P_{2} \leq \frac{C}{2\left|B_{0}\right|-M^{-}(t)}
$$

It follows that

$$
\int_{0}^{t} \frac{1}{\rho(\tau)^{\alpha}}\left|K\left(x^{-}(\tau), \tau\right)-K\left(y^{-}(\tau), \tau\right)\right| d \tau \leq \frac{C}{2\left|B_{0}\right|-M^{-}(t)} .
$$

Finally, in (4.10),

$$
\begin{aligned}
\alpha \int_{0}^{t} & \frac{\left|\rho^{\prime}(\tau)\right|}{\rho(\tau)^{\alpha+1}}\left|A^{+} j^{+}\left(x^{-}(\tau), \tau\right)-A^{+} j^{+}\left(y^{-}(\tau), \tau\right)\right| d \tau \\
& \leq \alpha \int_{0}^{\tau} \frac{\left|z^{+}\left(x^{-}(\tau), \tau\right)-z^{+}\left(y^{-}(\tau), \tau\right)\right|}{\rho(\tau)}\left|A^{+} j^{+}\left(x^{-}(\cdot), \tau\right)\right|_{0, \alpha} \\
& \leq \alpha \int_{0}^{t} \frac{1}{\rho(\tau)}\left|\frac{A^{-} z^{-}\left(x^{-}(\tau), \tau\right)}{f\left(x^{-}(\tau)-B_{0} \tau\right)}-\frac{A^{-} z^{-}\left(y^{-}(\tau), \tau\right)}{f\left(y^{-}(\tau)-B_{0} \tau\right)}\right|\left|A^{+} j^{+}(\cdot, \tau)\right|_{0, \alpha} d \tau
\end{aligned}
$$


and we proceed as above. We thus get the estimate

$$
\left|A^{+} j^{+}(\cdot, t)\right|_{0, \alpha} \leq\left|A^{+} j_{0}^{+}\right|_{0, \alpha}+\int_{0}^{\tau} G(\tau)\left[\left|A^{+} z^{+}(\cdot, \tau)\right|_{0, \alpha}+\left|A^{+} j^{+}(\cdot, \tau)\right|_{0, \alpha}\right] d \tau
$$

where

$$
\int_{0}^{t} G(\tau) d \tau \leq C \frac{M^{-}(t)}{2\left|B_{0}\right|-M^{-}(t)} .
$$

To estimate the sup norm of $A^{+} z^{+}(\cdot, \tau)$ (see (2.9)), the only new term to estimate is $\int_{0}^{t} A^{+} \nabla p\left(x^{-}(\tau), \tau\right) d \tau$. The pressure $p$ satisfies

$$
\Delta p=\operatorname{div}\left(z^{-} \cdot \nabla z^{+}\right)
$$

and is thus given by

$$
\partial_{i} p(x, t)=C_{n} \int_{R^{n}} \nabla\left[\frac{x_{i}-y_{i}}{|x-y|^{n}}\right] \cdot\left(z_{j}^{-} \partial_{j} z^{+}\right)(y) d^{n-1} y .
$$

The space dimension appears only in the kernel of the Poisson equation and in the element of integration. For simplicity we shall write the proof in the case of dimension three, since the extension to any other dimension $n \geq 2$ is straightforward. We adapt the analysis of the Poisson equation given by Ladyzhenskaya and Ural'tseva [5] (see also [6]), to take into account the weighted factors $f\left(x \pm B_{0} t\right)$.

$$
A^{+} \partial_{i} p(x, t)=\frac{1}{4 \pi} f\left(x+B_{0} t\right) \int_{R^{3}} \partial_{i} \nabla \frac{1}{|x-u|}\left(z_{j}^{-} \partial_{j} z^{+}\right)(u) d u .
$$

We separate the large and short distance contributions to the integral by writing

$$
A^{+} \partial_{i} p=\left(I_{1}+I_{2}\right) / 4 \pi
$$

with

$$
\begin{aligned}
& I_{1}(x) \equiv f\left(x+B_{0} t\right) \int_{R^{3}}\left(\partial_{i} \nabla \frac{\theta(|x-u|)}{|x-u|}\right)\left(z_{j}^{-} \partial_{j} z^{+}\right)(u) d u \\
& I_{2}(x) \equiv f\left(x+B_{0} t\right) \int_{R^{3}} \partial_{j} \partial_{i} \nabla \frac{1-\theta(|x-u|)}{|x-y|} z_{j}^{-} z^{+}(u) d u .
\end{aligned}
$$

$\theta(x)$ is the previously defined $C^{\infty}$ fucntion equal to 1 for $|x|<1$ and 0 for $|x|>2$. We rewrite $I_{1}$ in the form

$$
\begin{aligned}
& I_{1}=\frac{1}{f\left(x-B_{0} t\right)} \int d u \partial_{i} \nabla\left[\frac{\theta(|x-u|)}{|x-u|}\right] \\
& \cdot\left[\frac{f\left(x-B_{0} t\right)}{f\left(u-B_{0} t\right)} \frac{f\left(x+B_{0} t\right)}{f\left(u+B_{0} t\right)} A^{-} z_{j}^{-}(u, t) A^{+} \partial_{j} z^{+}(u, t)\right. \\
&\left.-A^{-} z_{j}^{-}(x, t) A^{+} \partial_{j} z^{+}(x, t)\right] .
\end{aligned}
$$

Thus

$$
\begin{aligned}
\left|I_{1}\right| \leq & \frac{C}{f\left(x-B_{0} t\right)}\left|A^{-} z^{-}(\cdot, t)\right|_{0, \alpha}\left|A^{+} \nabla z^{+}(\cdot, t)\right|_{0, \alpha} \\
& \cdot\left[1+\int_{|x-u|<1}|x-u|^{-3}\left[\frac{f\left(x-B_{0} t\right)}{f\left(u-B_{0} t\right)} \frac{f\left(x+B_{0} t\right)}{f\left(u+B_{0} t\right)}-1\right] d u\right] .
\end{aligned}
$$


We see that the integral in (4.27) is uniformly bounded by writing (4.28)

$$
\begin{aligned}
\frac{f(x-}{f\left(u-B_{0} t\right)} \frac{f\left(x+B_{0} t\right)}{f\left(u+B_{0} t\right)}-1 & \\
\quad= & -\frac{f\left(x+B_{0} t\right)\left(f\left(u-B_{0} t\right)-f\left(x-B_{0} t\right)\right)}{f\left(u+B_{0} t\right) f\left(u-B_{0} t\right)}+\frac{f\left(x+B_{0} t\right)-f\left(u+B_{0} t\right)}{f\left(u+B_{0} t\right)} \\
& \leq|x-u|\left[\frac{1+\left(x+B_{0} t\right)^{2}}{1+\left(u+B_{0} t\right)^{2}} \cdot \frac{\left|x+u-2 B_{0} t\right|}{1+\left(u-B_{0} t\right)^{2}}+\frac{x+u+2 B_{0} t}{1+\left(u+B_{0} t\right)^{2}}\right] \\
& \leq c|x-u| \quad \text { where }|x-u|<1 .
\end{aligned}
$$

Defining $a=\left|x+B_{0} t\right|$ and $b=\left|x-B_{0} t\right|$, we have

$$
\begin{aligned}
\left|I_{2}\right| \leq & \frac{C}{f\left(x-B_{0} t\right)}\left|A^{-} z^{-}(\cdot, t)\right|_{0}\left|A^{+} z^{+}(\cdot, \tau)\right|_{0} \\
& \cdot \int\left|\partial_{i} \partial_{j} \nabla\left[\frac{1-\theta(|u|)}{|u|}\right]\right| \frac{1+a^{2}}{1+\left(x+u+B_{0} t\right)^{2}} \frac{1+b^{2}}{1+\left(x+u-B_{0} t\right)^{2}} d u .
\end{aligned}
$$

The integral in (4.29) is bounded by the sum $J_{1}+J_{2}$ where

$$
J_{1}=C \int_{v>1} \frac{1}{v^{2}} \frac{1+a^{2}}{1+(v-a)^{2}} \cdot \frac{1+b^{2}}{1+(v-b)^{2}} d v
$$

and

$$
J_{2}=C \int_{1<v<2} \frac{1}{v} \frac{1+a^{2}}{1+(v-a)^{2}} \cdot \frac{1+b^{2}}{1+(v-b)^{2}}
$$

We have

$$
J_{1} \leq C\left[\int \frac{1}{v^{2}} \frac{\left(1+a^{2}\right)^{2}}{\left[1+(v-a)^{2}\right]^{2}} d v\right]^{1 / 2}\left[\int \frac{1}{v^{2}} \frac{\left(1+b^{2}\right)^{2}}{\left[1+(v-b)^{2}\right]^{2}} d v\right]^{1 / 2}
$$

$$
\begin{aligned}
\int \frac{\left(1+a^{2}\right)^{2} d v}{v^{2}\left[1+(v-a)^{2}\right]^{2}}= & \int_{v>1} \frac{d v}{v^{2}}+\frac{4 a}{1+a^{2}} \int\left[\frac{1}{v}+\frac{a-v}{1+(v-a)^{2}}\right] d v \\
& +\frac{3 a^{2}-1}{a^{2}+1} \int \frac{d v}{1+(v-a)^{2}}+\left(a^{2}-1\right) \int \frac{d v}{\left[1+(v-a)^{2}\right]^{2}} \\
& +2 a \int \frac{a-v}{\left[1+(v-s)^{2}\right]^{2}} d v \\
= & 1-\frac{4 a}{1+a^{2}} \ln \frac{1-a}{1+(1-a)^{2}}+\frac{3 a^{2}-1}{a^{2}+1} \tan ^{-1} \frac{1}{1-a} \\
& +\frac{1}{2}\left(a^{2}-1\right)\left[\tan ^{-1} \frac{1}{1-a}-\frac{1}{1-a} \frac{1}{1+1 /(1-a)^{2}}\right] \\
& +\frac{1}{1+(1-a)^{2}}
\end{aligned}
$$

is uniformly bounded from above in $a$. It follows that

$$
J_{1} \leq C \text {. }
$$


Proceeding similarly for $J_{2}$, we have

$$
J_{2} \leq C\left[\int_{1<v<2} \frac{\left(1+a^{2}\right)^{2} d v}{v\left[1+(v-a)^{2}\right]^{2}}\right]^{1 / 2}\left[\int_{1<v<2} \frac{\left(1+b^{2}\right)^{2} d v}{v\left[1+(v-b)^{2}\right]^{2}}\right]^{1 / 2} .
$$

Again

$$
\begin{aligned}
\int_{1<v<2} & \frac{\left(1+a^{2}\right)^{2} d v}{v\left[1+(v-a)^{2}\right]^{2}} \\
= & \int_{1<v<2} \frac{d v}{v}+\int \frac{(a-v)+a}{1+(v-a)^{2}} d v \\
& +\left(a^{2}+1\right) \int_{1<v<2} \frac{a-v+a}{\left[1+(v-a)^{2}\right]^{2}} d v \\
= & {\left[\ln \frac{v}{\sqrt{1+(v-a)^{2}}}\right]_{1}^{2}+\frac{3}{2} a\left[-\tan ^{-1}(v-a)\right]_{1}^{2} } \\
& +\frac{a^{2}+1}{2}\left[\frac{1}{1+(v-a)^{2}}+\frac{1}{2} \frac{v-a}{(v-a)^{2}+1}\right]_{1}^{2}
\end{aligned}
$$

is uniformly bounded in $a$. Thus, in (2.17)

$$
\begin{aligned}
\left|\int_{0}^{t} A^{+} \nabla p\left(x^{-}(\tau), \tau\right) d \tau\right| \leq C & \int_{0}^{t} \frac{d \tau}{f\left(x^{-}(\tau)-B_{0} \tau\right)}\left|A^{-} z^{-}(\cdot, \tau)\right|_{0, \alpha} \\
& \cdot\left[\left|A^{+} z^{+}(\cdot, \tau)\right|_{0, \alpha}+\left|A^{+} \nabla z^{+}(\cdot, \tau)\right|_{0, \alpha}\right]
\end{aligned}
$$

and, consequently,

$$
\left|A^{+} z^{+}(\cdot, t)\right|_{0} \leq\left|A^{+} z_{0}^{+}\right|_{0}+\int_{0}^{t} G(\tau)\left[\left|A^{+} z^{+}(\cdot, \tau)\right|_{0, \alpha}+\left|A^{+} j^{+}(\cdot, \tau)\right|_{0, \alpha}\right] d \tau
$$

where

$$
\int_{0}^{t} G(\tau) d \tau \leq C \frac{M^{-}(t)}{2\left|B_{0}\right|-M^{-}(t)}
$$

Let us now turn to the $C^{0, \alpha}$ norm of $A^{+} z^{+}$. As in (4.9), (4.10), we have

$$
\begin{aligned}
& \frac{\left|A^{+} z^{+}\left(x^{-}(t), t\right)-A^{+} z^{+}\left(y^{-}(t), t\right)\right|}{\rho(t)^{\alpha}} \\
& \quad \leq\left|A^{+} z_{0}^{+}\right|_{0, \alpha}+\alpha \int_{0}^{t} \frac{\left|\rho^{\prime}(\tau)\right|}{\rho(\tau)^{\alpha+1}}\left|A^{+} z^{+}\left(x^{-}(\tau), \tau\right)-A^{+} z^{+}\left(y^{-}(\tau), \tau\right)\right| d \tau \\
& \quad+\int_{0}^{t} \frac{1}{\rho(\tau)^{\alpha}}\left|A^{+} \nabla p\left(x^{-}(\tau), \tau\right)-A^{+} \nabla p\left(y^{-}(\tau), \tau\right)\right| d \tau .
\end{aligned}
$$

For simplicity, we write $x \equiv x^{-}(\tau)$ and $y \equiv y^{-}(\tau), \rho \equiv|x-y|$. Using the notations of $(4.24)$ and (4.25) we have

$$
\begin{aligned}
& \frac{1}{\rho^{\alpha}}\left(A^{+} \nabla p(x, \tau)-A^{+} \nabla p(y, \tau)\right) \\
& \quad=\frac{1}{\rho^{\alpha}}\left(I_{1}(x)-I_{1}(y)\right)+\frac{1}{\rho^{\alpha}}\left(I_{2}(x)-I_{2}(y)\right) .
\end{aligned}
$$

For $\rho<\frac{1}{2}$, in the expression of $\left(I_{1}(x)-I_{1}(y)\right) / \rho^{\alpha}$, we separate the contributions $\sigma$ from $|x-u|<2 \rho$ and $\bar{\sigma}$ from $|x-u|>2 \rho$. 

that

$$
|y-u| \leq|x-u|+|x-y| \leq 3 \rho .
$$

Thus, proceeding as in (4.26)-(4.29), we obtain

$$
\int_{0}^{t} \sigma d \tau \leq \int_{0}^{t} G(\tau)\left[\left|A^{+} z^{+}(\cdot, \tau)\right|_{0, \alpha}+\left|A^{+} j^{+}(\cdot, \tau)\right|_{0, \alpha}\right] d \tau
$$

with $G$ satisfying (4.39).

Turning now to $\bar{\sigma}$, we have

$$
\bar{\sigma}=K_{1}+K_{2}+K_{3}
$$

with

$$
\begin{aligned}
& K_{1}= \frac{1}{\rho^{\alpha}}\left[\frac{1}{f\left(x-B_{0} t\right)}-\frac{1}{f\left(y-B_{0} t\right)}\right] \\
& \cdot \int \partial_{i} \nabla\left[\frac{\theta(|x-u|)}{|x-u|}\right]\left[\frac{f\left(x-B_{0} t\right)}{f\left(u-B_{0} t\right)} \frac{f\left(x+B_{0} t\right)}{f\left(u+B_{0} t\right)} A^{-} z_{j}^{-}(u, t) A^{+} \partial_{j} z^{+}(u, t)\right. \\
&\left.-A^{-} z_{j}^{-}(x, t) A^{+} \partial_{j} z^{+}(x, t)\right] d u
\end{aligned}
$$

$$
\begin{aligned}
& K_{2}=\frac{1}{\rho^{\alpha}} \frac{1}{f\left(y-B_{0} t\right)} \int {\left[\partial_{i} \nabla \frac{\theta(|x-u|)}{|x-u|}-\partial_{i} \nabla \frac{\theta(|y-u|)}{y-u \mid}\right] } \\
& \cdot\left[\frac{f\left(x-B_{0} t\right)}{f\left(u-B_{0} t\right)} \frac{f\left(x+B_{0} t\right)}{f\left(u+B_{0} t\right)} A^{-} z_{j}^{-}(u, t) A^{+} \partial_{j} z^{+}(u, t)\right. \\
&\left.-A^{-} z_{j}^{-}(x, t) A^{+} \partial_{j} z^{+}(x, t)\right] d u,
\end{aligned}
$$

$$
\begin{aligned}
K_{3}=\frac{1}{\rho^{\alpha} f\left(x-B_{0} t\right)} \int & \partial_{i} \nabla\left[\frac{\theta(|y-u|)}{|y-u|}\right] \\
& \cdot \frac{\left(f\left(x-B_{0} t\right) f\left(x+B_{0} t\right)-f\left(y-B_{0} t\right) f\left(y+B_{0} t\right)\right)}{f\left(u-B_{0} t\right) f\left(u+B_{0} t\right)} \\
& \cdot A^{-} z_{j}^{-}(u, t) A^{+} \partial_{j} z^{+}(u, t) \\
& -\left[A^{-} z_{j}^{-}(x, t) A^{+} \partial_{j} z^{+}(x, t)-A^{-} z_{j}^{-}(y, t) A^{+} \partial_{j} z^{+}(y, t)\right] d u .
\end{aligned}
$$

In $K_{1}$ we have

$$
\rho^{-\alpha}\left[\frac{1}{f\left(x-B_{0} t\right)}-\frac{1}{f\left(y-B_{0} t\right)}\right] \leq C \rho^{1-\alpha}\left[\frac{1}{f\left(x-B_{0} t\right)}+\frac{1}{f\left(y-B_{0} t\right)}\right] .
$$

Thus, using (4.28), we have

$$
K_{1} \leq \int_{0}^{t} G(\tau)\left(\left|A^{+} z^{+}(\cdot, \tau)\right|_{0, \alpha}+\left|A^{+} j^{+}(\cdot, \tau)\right|_{0, \alpha}\right) d \tau
$$


with $G$ satisfying (4.39). For $K_{2}$, we write

$$
\partial_{i} \nabla \frac{\theta(|x-u|)}{|x-u|}-\partial_{i} \nabla \frac{\theta(|y-u|)}{|y-u|} \leq C|x-y| \sup _{0 \leq \eta \leq 1}|u-\eta y-(1-\eta) x|^{-4}
$$

but

$$
\begin{aligned}
|u-\eta y-(1-\eta) x| & \geq|u-x|-\eta|x-y| \\
& \geq|u-x|-\frac{\eta}{2}|u-x|>\frac{1}{2}|u-x| .
\end{aligned}
$$

Thus

$$
\begin{aligned}
K_{2} \leq & \frac{1}{f\left(y-B_{0} t\right)} \rho^{1-\alpha}\left|A^{-} z^{-}(\cdot, t)\right|_{0, \alpha}\left|A^{+} \nabla z^{+}(\cdot, t)\right|_{0, \alpha} \\
& \cdot \int_{2 \rho<|u-x|<1} \frac{1}{|x-u|^{4-\alpha}} d u \\
& +\int_{2 \rho<|x-u|<1} \frac{1}{|x-u|^{4}}\left[\frac{f\left(x-B_{0} t\right)}{f\left(u-B_{0} t\right)} \frac{f\left(x+B_{0} t\right)}{f\left(u+B_{0} t\right)}-1\right] d u
\end{aligned}
$$

In (4.52)

$$
\int_{2 \rho<|u-x|<1} \frac{1}{|x-u|^{4-\alpha}} d u \leq C\left(\rho^{\alpha-1}-1\right)
$$

and

$$
\begin{gathered}
\int_{2 \rho<|u-x|<1} \frac{1}{|x-u|^{4}}\left[\frac{f\left(x-B_{0} t\right)}{f\left(u-B_{0} t\right)} \frac{f\left(x+B_{0} t\right)}{f\left(u+B_{0} t\right)}-1\right] d u \\
\leq C \int_{2 \rho<|u-x|<1} \frac{1}{|x-u|^{3}} d u \leq C \ln \frac{1}{2 \rho} .
\end{gathered}
$$

Putting together the above estimates, we get

$$
K_{2}=\frac{C}{f\left(y-B_{0} t\right)}\left|A^{-} z^{-}(\cdot, t)\right|_{0, \alpha}\left|A^{+} \nabla z^{+}(\cdot, t)\right|_{0, \alpha} .
$$

Finally, in the expression (4.47) of $K_{3}$, we have

$$
\begin{aligned}
\left|f\left(x-B_{0} t\right) f\left(x+B_{0} t\right)-f\left(y-B_{0} t\right) f\left(y+B_{0} t\right)\right| \\
\leq|x-y|\left[\left[1+\left(x+B_{0} t\right)^{2}\right]\left[x+y-2 B_{0} t\right]\right. \\
\left.+\left[1+\left(y-B_{0} t\right)^{2}\right]\left[x+y+2 B_{0} t\right]\right] .
\end{aligned}
$$

Thus

$$
\begin{aligned}
& \frac{1}{\rho^{\alpha}} \int \theta(|y-u|) \partial_{i} \nabla \frac{1}{|y-u|} \\
& \cdot\left[\frac{f\left(x-B_{0} t\right) f\left(x+B_{0} t\right)-f\left(y-B_{0} t\right) f\left(y+B_{0} t\right)}{f\left(u-B_{0} t\right) f\left(u+B_{0} t\right)}\right] d u \\
& \leq C \rho^{1-\alpha} \int_{\rho<|y-u|<1} \frac{1}{|y-u|^{3}} d u=C \rho^{1-\alpha} \ln \frac{1}{\rho} \leq C^{\prime}
\end{aligned}
$$

and

$$
K_{3} \leq \frac{C}{f\left(y-B_{0} t\right)}\left|A^{-} z^{-}(\cdot, t)\right|_{0, \alpha}\left|A^{+} \nabla z^{+}(\cdot, t)\right|_{0, \alpha}
$$


Putting together (4.49), (4.55), (4.58), we have

$$
\int_{0}^{t} \bar{\sigma} d \tau \leq \int_{0}^{t} G(\tau)\left(\left|A^{+} z^{+}(\cdot, \tau)\right|_{0, \alpha}+\left|A^{+} \nabla z^{+}(\cdot, \tau)\right|_{0, \alpha}\right) d \tau
$$

with $G$ satisfying (4.39).

Turning now to the second term of $(4.41)$, we write, when $\rho(t)<1$,

$$
\frac{1}{\rho(\tau)^{\alpha}}\left|I_{2}\left(x^{-}(\tau), \tau\right)-I_{2}\left(y^{-}(\tau), \tau\right)\right|=J_{1}+J_{2}
$$

with

$$
\begin{aligned}
J_{1}= & \frac{1}{\rho(t)^{\alpha}} \frac{\left|f\left(x^{-}(t)+B_{0} t\right)-f\left(y^{-}(t)+B_{0} t\right)\right|}{f\left(x^{-}(t)-B_{0} t\right) f\left(x^{-}(t)+B_{0} t\right)} \\
& \int \partial_{j} \partial_{i} \nabla \frac{1-\theta\left(\left|x^{-}(t)-u\right|\right)}{\left|x^{-}(t)-u\right|} \\
& \frac{A^{-} z^{-}(u) A^{+} \nabla z^{+}(u) f\left(x^{-}(t)+B_{0} t\right) f\left(x^{-}(t)-B_{0} t\right)}{f\left(u+B_{0} t\right) f\left(u-B_{0} t\right)} d u
\end{aligned}
$$

and

$$
\begin{aligned}
J_{2}= & \frac{1}{\rho(t)^{\alpha}}\left(f\left(y^{-}(t)+B_{0} t\right)\right) \int \partial_{j} \partial_{i} \nabla \frac{1-\theta(|x(t)-u|)}{\left|x^{-}(t)-u\right|} \\
& -\partial_{j}(1-\theta(|y(t)-y|)) \partial_{i} \nabla \frac{1}{\left|y^{-}(t)-u\right|} \frac{A^{-} z^{-}(u) A^{+} \nabla z^{+}(u)}{f\left(u+B_{0} t\right) f\left(u-B_{0} t\right)} d u
\end{aligned}
$$

We have

$$
J_{1}=\frac{\rho(t)^{1-\alpha}}{f\left(x^{-}(t)-B_{0} t\right)}\left|A^{-} z^{-}(\cdot, t)\right|_{0}\left|A^{+} \nabla z^{+}(\cdot, t)\right|_{0} .
$$

For $J_{2}$, we write $(w=\gamma x+(1-\gamma) y, 0<\gamma<1)$

$$
\begin{gathered}
J_{2} \leq \rho(t)^{1-\alpha} f\left(y^{-}(t)+B_{0} t\right) \\
\cdot \int \nabla \nabla\left[\frac{1-\theta(|w-u|)}{|w-u|^{3}}\right] \frac{A^{-} z^{-}(u)}{f\left(u-B_{0} t\right)} \frac{A^{+} z^{+}(u)}{f\left(u+B_{0} t\right)} d u \\
\leq \rho(t)^{1-\alpha} \frac{1}{f\left(y^{-}(t)-B_{0} t\right)}\left|A^{-} z^{-}(\cdot, t)\right|_{0}\left|A^{+} z^{+}(\cdot, t)\right|_{0} \\
\cdot \int \nabla \nabla\left[\left(1-\frac{\theta(|w-u|)}{|w-u|^{3}}\right)\right] \\
\cdot \frac{f\left(w-B_{0} t\right) f\left(w+B_{0} t\right)}{f\left(u-B_{0} t\right) f\left(u+B_{0} t\right)} \cdot \frac{f\left(y-B_{0} t\right) f\left(y+B_{0} t\right)}{f\left(w-B_{0} t\right) f\left(w+B_{0} t\right)} d u .
\end{gathered}
$$

In (4.65)

$$
\frac{f\left(y-B_{0} t\right) f\left(y+B_{0} t\right)}{f\left(w-B_{0} t\right) f\left(w+B_{0} t\right)}<C
$$

because $w=y+\gamma(x-y)$ and $|x-y|=\rho<1$, and

(4.66) $\nabla \nabla\left[\frac{1-\theta(|w-u|)}{|w-u|^{3}}\right] \leq C\left[\frac{1-\theta(|w-u|)}{|w-u|^{5}}+\frac{\theta^{\prime}(|w-u|)}{|w-u|^{4}}+\frac{\theta^{\prime \prime}(|w-u|)}{|w-u|^{3}}\right]$. 
Thus,

$$
\begin{aligned}
\int \nabla \nabla & {\left[\frac{1-\theta(|w-u|)}{|w-u|^{3}}\right] \frac{f\left(w-B_{0} t\right) f\left(w+B_{0} t\right)}{f\left(u-B_{0} t\right) f\left(u+B_{0} t\right)} d u } \\
\leq & \int_{|w-u|>1} \frac{1}{|w-u|^{4}} \frac{f\left(w-B_{0} t\right) f\left(w+B_{0} t\right)}{\left(f\left(u-B_{0} t\right)\right) f\left(u+B_{0} t\right)} d u \\
& +\int_{1<|w-u|<2} \frac{1}{|w-u|^{3}} \frac{\left(f\left(w-B_{0} t\right) f\left(w+B_{0} t\right)\right.}{\left(f\left(u-B_{0} t\right)\right) f\left(u+B_{0} t\right)} d u .
\end{aligned}
$$

These last expressions are uniformly bounded in $x, y, t$ (see (4.28)). Thus,

$$
J_{2} \leq \frac{C}{f\left(y^{-}(t)-B_{0} t\right)}\left|A^{-} z^{-}(\cdot, t)\right|_{0}\left|A^{+} z^{+}(\cdot, t)\right|_{0} .
$$

Putting all the estimates together we obtain

$$
\begin{aligned}
& \frac{1}{\rho(\tau)^{\alpha}}\left|A^{+} \nabla p\left(x^{-}(\tau)+B_{0} \tau\right)-A^{+} \nabla p\left(y^{-}(\tau)+B_{0} \tau\right)\right| \\
& \quad \leq \frac{C}{f\left(y^{-}(\tau)-B_{0} \tau\right)}\left|A^{-} z^{-}(\cdot, \tau)\right|_{0, \alpha}\left(\left|A^{+} z^{+}(\cdot, \tau)\right|_{0, \alpha}+\left|A^{+} \nabla z^{+}(\cdot, \tau)\right|_{0, \alpha}\right) .
\end{aligned}
$$

The second integral in (2.9) is in the form of that appearing in (2.8). We finally get

$$
\begin{aligned}
& \left|A^{+} z^{+}(\cdot, t)\right|_{0, \alpha} \\
& \quad \leq\left|f z^{+}\right|_{0, \alpha}+\int_{0}^{t} G(\tau)\left(\left|A^{+} z^{+}(\cdot, \tau)\right|_{0, \alpha}+\left|A^{+} j^{+}(\cdot, \tau)\right|_{0, \alpha}\right) d \tau
\end{aligned}
$$

with $G$ satisfying (4.39).

We obtain estimate (4.19) using Gronwall's lemma.

PROPOSITION 4.2. There exists a positive constant $\beta$ such that if the initial conditions satisfy

$$
\delta \equiv\left|f z_{0}^{+}\right|_{1, \alpha}+\left|f z_{0}^{-}\right|_{1, \alpha} \leq \beta\left|B_{0}\right|,
$$

the quantities $M^{ \pm}(T)\left(T<T_{1}\right)$ remain bounded independently of $T$.

ProOF. From Proposition 4.1, we have

$$
N(T) \leq \delta \exp \frac{C N(T)}{2\left|B_{0}\right|-N(T)}
$$

We define

$$
g(x)=\delta \exp \frac{C x}{2\left|B_{0}\right|-x}-x
$$

Introducing $\eta=1 /\left(2\left|B_{0}\right|-x\right)$, we rewrite $g$ as

$$
g(x)=h(\eta)=\delta \bar{e}^{C} e^{2 C\left|B_{0}\right| \eta}-2 B_{0}+1 / \eta,
$$

and

$$
h^{\prime}(\eta)=2 C \bar{e}^{C} \delta\left|B_{0}\right| e^{2 C\left|B_{0}\right| \eta}-1 / \eta^{2}
$$


vanishes at $\eta_{*}$, which is given by

$$
\eta_{*}^{2} e^{2 C\left|B_{0}\right| \eta *}=e^{C} / 2 C \delta\left|B_{0}\right| .
$$

The value of $h$ at that point is given by

$$
h\left(\eta_{*}\right)=\frac{1}{2 C\left|B_{0}\right|} \frac{1}{\eta_{*}^{2}}-2 B_{0}+\frac{1}{\eta_{*}} .
$$

The condition $h\left(\eta_{*}\right)<0$ leads to

$$
\eta_{*}>\frac{1}{C\left|B_{0}\right|} \frac{1}{-1+\sqrt{1+4 / C}}
$$

Substitution in (2.81) gives

$$
\frac{e^{C}}{2 C \delta\left|B_{0}\right|}>\frac{1}{\left(C B_{0}\right)^{2}}\left[\frac{1}{-1+\sqrt{1+4 / C}}\right]^{2} \exp \frac{2}{-1+\sqrt{1+4 / C}}
$$

or

$$
\delta /\left|B_{0}\right|<\beta_{1}
$$

for a certain positive constant $\beta_{1}$. Under this condition $g$ has at least one zero $x_{1}=\delta \exp C x_{1} /\left(2\left|B_{0}\right|-x_{1}\right)$. Thus $\delta<x_{1}$. Now, if the initial conditions are such that $\delta<\beta\left|B_{0}\right|\left(\beta=\min \left(1 / 4, \beta_{1}\right)\right)$, then $N(T)$ will remain smaller than $x_{1}$ for all $T<T_{1}$ independently of $T$.

\section{Existence in the large and asymptotic freedom.}

THEOREM 5.1. There exists a constant $\beta$ such that, if the intial conditions satisfy

$$
\left|f z_{0}^{+}\right|_{1, \alpha}+\left|f z_{0}^{+}\right|_{1, \alpha}<\beta\left|B_{0}\right|,
$$

there exists for all time a solution $\left(z^{+}, z^{-}\right)$in $C\left(R^{+}, C^{1, \alpha}\left(R^{n}\right)\right)$. Moreover

$$
\left|A^{ \pm} z^{ \pm}(\cdot, t)\right|_{0, \alpha}+\left|A^{ \pm} \nabla z^{ \pm}(\cdot, t)\right|_{0, \alpha}
$$

is uniformly bounded.

ProOF. During the time interval $\left[0, T_{1}\right]$ when the existence theorem holds, we have, using the above proposition,

$$
\left|z^{ \pm}(\cdot, t)\right|_{0, \alpha} \leq\left|A^{ \pm} z^{ \pm}(\cdot, \alpha) 1 / f\right|_{0, \alpha} \leq C K
$$

and

$$
\left|\nabla z^{ \pm}(\cdot, t)\right|_{0, \alpha} \leq\left|A^{ \pm} \nabla z^{ \pm}(\cdot, \alpha) 1 / f\right|_{0, \alpha} \leq C K .
$$

We then repeatedly use the local existence theorem.

THEOREM 5.2. Under conditions of Theorem 5.1, the solution $\left(z^{+}, z^{-}\right)$of $(2.1)$ tends to a solution of the linear problem

$$
\frac{\partial}{\partial t} w^{ \pm} \mp B_{0} \nabla w^{ \pm}=0
$$

when $t \rightarrow+\infty$. 
Proof. We define

$$
w^{ \pm}(x, t)=z^{ \pm}(x, t)-\int_{t}^{+\infty}\left(z^{\mp} \cdot \nabla z^{ \pm}+\nabla p\right)\left(x \mp B_{0}(t-\tau), \tau\right) d \tau .
$$

Since

$$
\begin{aligned}
\mid \int_{0}^{+\infty} & \left(z^{-} \nabla z^{+}+\nabla p\right)\left(x+B_{0}(t-\tau), \tau\right) d \tau \mid \\
\leq & \left|A^{-} z^{-}\right|_{0, \alpha}\left[\left|A^{+} z^{+}\right|_{0, \alpha}+\left|A^{+} \nabla z^{+}\right|_{0, \alpha}\right] \\
& \cdot \frac{1}{1+\left(x+B_{0} t\right)^{2}} \int_{0}^{\infty} \frac{d \tau}{1+\left(x+B_{0}(t-2 \tau)\right)^{2}} \\
\leq & \frac{C}{\left|B_{0}\right|} \frac{1}{1+\left(x+B_{0} t\right)^{2}}
\end{aligned}
$$

(and a similar estimate when the + and - are exchanged), $\left|w^{ \pm}(x, t)-z^{ \pm}(x, t)\right|$ tends to zero pointwise when $t \rightarrow+\infty$. Furthermore $w^{ \pm}$satisfies (5.3).

ACKNOWLEDGMENT. We thank S. Klainerman for useful discussions.

\section{REFERENCES}

1. R. H. Kraichnan, Phys. Fluids 8 (1965), 1385.

2. U. Frisch, A. Pouquet, P. L. Sulem and M. Meneguzzi, J. Méc. Théor. Appl., Special issue on two dimensional turbulence, 1983, pp. 191-216.

3. S. Klainerman, Comm. Pure Appl. Math. 36 (1983), 325-344.

4. C. Sulem, C. R. Acad Sci. Paris Ser. A 285 (1977), 365-368.

5. O. A. Ladyzenskaya and N. N. Ural'tseva, Linear and quasilinear elliptic equations, Math. in Science and Engineering, vol. 46, Academic Press, 1968.

6. C. Bardos and U. Frisch, Turbulence and Navier-Stokes equations (Ed., R. Temam), Lecture Notes in Math., vol. 565, Springer-Verlag, 1976, p. 1.

7. S. Klainerman and G. Ponce, Comm. Pure Appl. Math. 33 (1980), 43-101.

Département de Mathématiques, Université de Paris XIII and Centre de Mathématiques Appliquées, ECole Normale Supérieure, Paris, France

Department of Mathematics, Ben Gurion University of the Negev, Beer Sheva, IsRael and CNRS, Centre de Mathématiques APpliquées, ECole Normale SUPÉRIEURE, PARIS, FRANCE

School of Mathematical Sciences, Tel-Aviv University, Israel and CNRS, OBSERVATOIRE DE Nice, FRANCE 